\title{
Parameter Driven Modeling of Planar Double-enveloping Toroid Worm Pair
}

\author{
Zhi LIU ${ }^{1,} a^{*}$, Hong LU², Guo-Ming YU ${ }^{1}$ \\ ${ }^{1}$ School of Mechanical and Electronic Engineering, Huanggang Normal University, Huanggang \\ 438000, China \\ ${ }^{2}$ School of Mechanical and Electronic Engineering, Wuhan University of Technology, Wuhan \\ 430070, China \\ aIz3839@126.com \\ ${ }^{*}$ Corresponding author
}

Keywords: Toroid Worm Pair, Parameter Driven, Meshing Surface, Digital Model, MATLAB.

\begin{abstract}
The parameters calculation, meshing surface and $3 \mathrm{D}$ models construction of planar double-enveloping toroid worm pair are efforts consuming because of complexity of the envelope motion. A parameter driven modeling method of this worm pair has been proposed, the parameters calculation, meshing surface and 3D models construction of the worm pair are connected with basic parameters applied embedded method, all of the parameters, meshing surface and $3 \mathrm{D}$ models could be obtained driven by the basic parameters, in addition, the parameter driven modeling system is developed applied MATLAB. The experiment results indicate that rapid parameter driven modeling could be achieved of any planar double-enveloping toroid worm pair according to the proposed modeling method and parameter driven modeling system, including parameters calculation, surface presentation and models construction.
\end{abstract}

\section{Introduction}

Due to the singularity of the enveloping movement and flexibility of envelope trajectory, planar double-enveloping toroid worm pair has the characteristics of double contact lines, multi-meshing teeth at the same time, as a result, this kind of worm pair has the advantiges of compact transmission, strong carrying capacity, long life and so on[1-2], they are widely applied in navigation, aviation, energy, industry, and other related areas. However, the parameters calculation, surface representation and accurate $3 \mathrm{D}$ modeling are very complicated because of complexity of the envelope motion. In addition, this kind worm pair have wide range of center distance and transmission ratio, so parameters calculation and model construction are usually low efficiency, poor precision and high professional requirements.

The mathematical study was conducted of planar double-enveloping toroid worm pair, the original parameters of tooth surface for this worm pair are obtained, and the entity model built methods applied 3D modeling software was discussed[3-4]. The 3D entity model was built of this worm pair using 3D modelling software, and virtual manufacturing study was also conducted on this basis the models[5]. The tooth surface of planar double-enveloping toroid worm pair are non developable surface, so the 3D modelling software couldn't obtain high accuracy model, the model and manufacturing precision still need to improve.

Parameters optimization design was carried out for planar double-enveloping toroid worm pair considering lubrication effect, and relevant software interface was developed using VB[6-7]. But it cann't obtain all contact lines and 3D model for this worm pair according to the developed software interface.

Aims at improving calculation accuracy and modeling precision as well as efficiency, a parameter driven modeling method for planar double-enveloping toroid worm pair is proposed in this paper. 


\section{Parameter Driven Modeling Method}

Different from ordinary worm drive, the tooth surface of planar double-enveloping toroid worm pair is enveloped by the envelope motion, their surface is non developable surface as well as complicate surface, and the calculation parameters are various, as a result, the parameters calculation and model construction are efforts consuming. On the other hand, the parameters calculation and model construction could obtained according to center distance, transmission ratio and threads of the worm, this provides the foundation and feasibility for parameter driven modeling for this worm pair.

The conception of parameter driven modeling method is connect parameters calculation and modeling with center distance, transmission ratio and threads of the worm, so as to simplify parameters calculation and modeling process as well as improve design efficiency and accuracy.

\section{Parameter Driven Model}

The mathematical system of planar double-enveloping toroid worm pair is constructed as below:

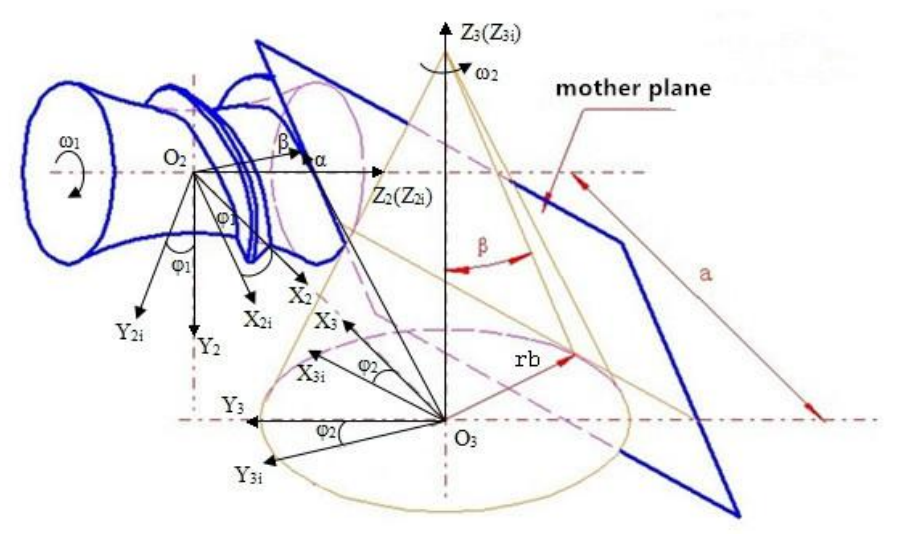

Fig.1 The mathematical modeling system for the worm pair

The mathematical model of the worm surface is represent as follows[8]:

$$
\left\{\begin{aligned}
\vec{n} * \overrightarrow{v_{12}} & =0 \\
{\left[\begin{array}{l}
x_{1} \\
y_{1} \\
z_{1}
\end{array}\right]=} & {\left[\begin{array}{ccc}
1 & 0 & 0 \\
0 & \sin \beta_{0} & -\cos \beta_{0} \\
0 & \cos \beta_{0} & \sin \beta_{0}
\end{array}\right]\left[\begin{array}{l}
u \\
v \\
0
\end{array}\right] } \\
{\left[\begin{array}{l}
x_{2} \\
y_{2} \\
z_{2}
\end{array}\right]=} & \left\{\left[\begin{array}{ccc}
\cos \varphi_{2} & -\sin \varphi_{2} & 0 \\
\sin \varphi_{2} & \cos \varphi_{2} & 0 \\
0 & 0 & 1
\end{array}\right]\left[\begin{array}{lll}
1 & 0 & 0 \\
0 & 0 & -1 \\
0 & 1 & 0
\end{array}\right]\left[\begin{array}{ccc}
-1 & 0 & 0 \\
0 & -1 & 0 \\
0 & 0 & 1
\end{array}\right]\left[\begin{array}{l}
x_{1} \\
y_{1} \\
z_{1}
\end{array}\right]+\left[\begin{array}{l}
-a \\
0 \\
0
\end{array}\right]\right\} \\
& *\left[\begin{array}{ccc}
\cos \varphi_{1} & -\sin \varphi_{1} & 0 \\
\sin \varphi_{1} & \cos \varphi_{1} & 0 \\
0 & 0 & 1
\end{array}\right]
\end{aligned}\right.
$$

The mathematical model of the worm wheel surface is represent as follows[8]: 


$$
\begin{aligned}
& {\left[\begin{array}{l}
x_{1} \\
y_{1} \\
z_{1}
\end{array}\right]=} {\left[\begin{array}{ccc}
1 & 0 & 0 \\
0 & \sin \beta_{0} & -\cos \beta_{0} \\
0 & \cos \beta_{0} & \sin \beta_{0}
\end{array}\right]\left[\begin{array}{l}
u \\
v \\
0
\end{array}\right] } \\
& {\left[\begin{array}{l}
x_{2} \\
y_{2} \\
z_{2}
\end{array}\right]=}\left.\left\{\begin{array}{ccc}
\cos \varphi_{2} & -\sin \varphi_{2} & 0 \\
\sin \varphi_{2} & \cos \varphi_{2} & 0 \\
0 & 0 & 1
\end{array}\right]\left[\begin{array}{lll}
1 & 0 & 0 \\
0 & 0 & -1 \\
0 & 1 & 0
\end{array}\right]\left[\begin{array}{lll}
-1 & 0 & 0 \\
0 & -1 & 0 \\
0 & 0 & 1
\end{array}\right]\left[\begin{array}{l}
x_{1} \\
y_{1} \\
z_{1}
\end{array}\right]+\left[\begin{array}{l}
-a \\
0 \\
0
\end{array}\right]\right\} \\
&\left.*\left[\begin{array}{ccc}
\cos \varphi_{1}-\sin \varphi_{1} & 0 \\
\sin \varphi_{1} & \cos \varphi_{1} & 0 \\
0 & 0 & 1
\end{array}\right]\right\} \\
& {\left.\left[\begin{array}{l}
x_{1} \\
y_{1} \\
z_{1}
\end{array}\right]=\left\{\begin{array}{ccc}
\cos \varphi_{2}^{\prime} & \sin \varphi_{2}^{\prime} & 0 \\
-\sin \varphi_{2}^{\prime} & \cos \varphi_{2}^{\prime} & 0 \\
0 & 0 & 1
\end{array}\right]\left[\begin{array}{lll}
1 & 0 & 0 \\
0 & 0 & 1 \\
0 & -1 & 0
\end{array}\right]\left[\begin{array}{lll}
-1 & 0 & 0 \\
0 & -1 & 0 \\
0 & 0 & 1
\end{array}\right]\left[\begin{array}{l}
x_{2} \\
y_{2} \\
z_{2}
\end{array}\right]+\left[\begin{array}{l}
a \\
0 \\
0
\end{array}\right]\right\} } \\
& {\left.\left[\begin{array}{ccc}
\cos \varphi_{1}^{\prime} & \sin \varphi_{1}^{\prime} & 0 \\
-\sin \varphi_{1}^{\prime} & \cos \varphi_{1}^{\prime} & 0 \\
0 & 0 & 1
\end{array}\right]\right\} }
\end{aligned}
$$

Where, $a$ represent center distance, $\varphi$ represent envelope angle, $\beta_{0}$ represent tilt angle, $[u, v, 0]^{T}$ indicates a vector that represents the mother plane surface, $\left[x_{2}, y_{2}, z_{2}\right]^{T}$ indicates a vector that represents the worm wheel surface, $\left[x_{1}, y_{1}, z_{1}\right]^{T}$ indicates a vector that represents the worm surface, $n$ states the normal vector in mother plane, $v_{12}$ states the relative velocity vector of the mother plane and the work-piece.

\section{Parameter Driven Modeling System}

The parameter driven modeling system is developed by MATLAB in this section, and the develope flowchat is given as below(Fig.2):

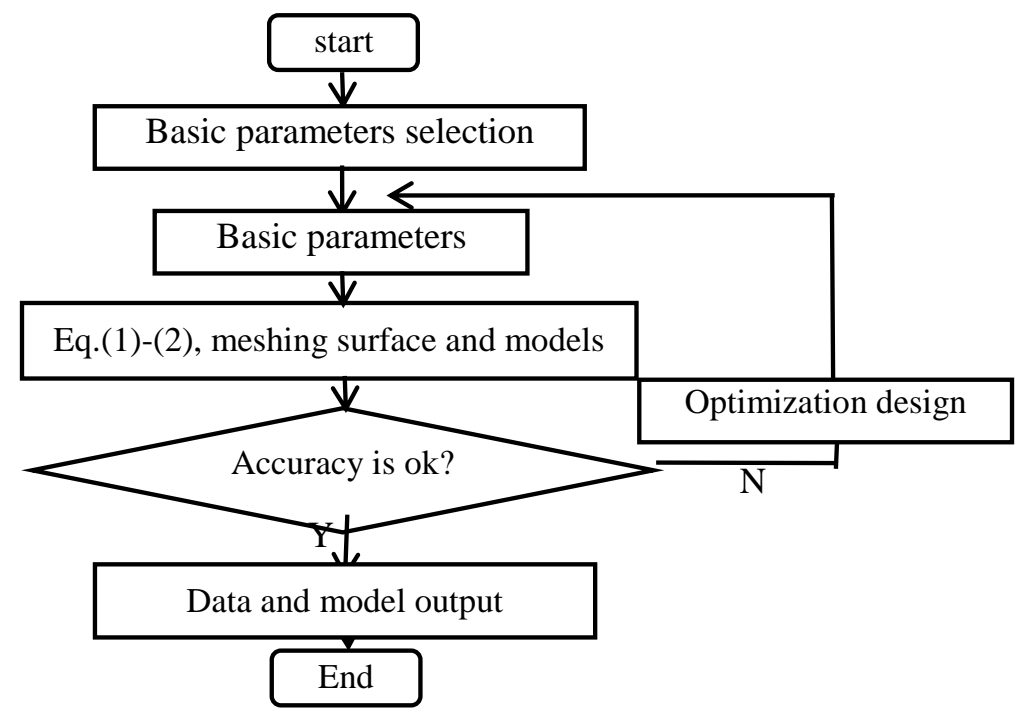

Fig.2 The flowchart of parameter driven modeling system

The developed parameter driven modeling interface is show as Fig.3.

\section{Parameter Driven Modeling Experiment}

To validate the parameter driven modeling method, a planar double-enveloping toroid worm pair modeling example are carried out in this section. The basic parameters are list in Tab.1. 
Tab.1 The basic parameters of the worm pair

\begin{tabular}{lll}
\hline Item & Symbol/unit & Value \\
\hline Center distance & $a / m m$ & 140 \\
$\begin{array}{l}\text { Number of the threads of the worm having } \\
\text { right-handed helix }\end{array}$ & $z_{1}$ & 1 \\
The transmission ratio & $i_{12}$ & 40 \\
\hline
\end{tabular}

The geometry and modeling parameters, worm surface and model, wheel surface and model are obtained rpidly according to the developed modeling system(Fig.3 to Fig.5).

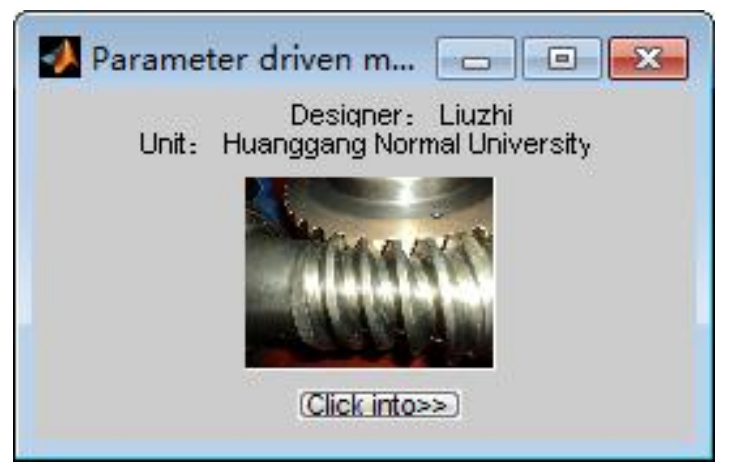

Fig.3 The parameter driven modeling system

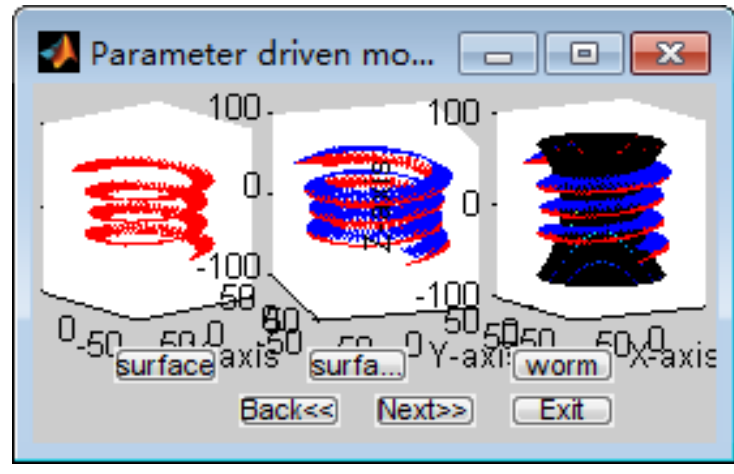

Fig.4 The surface and model of the worm

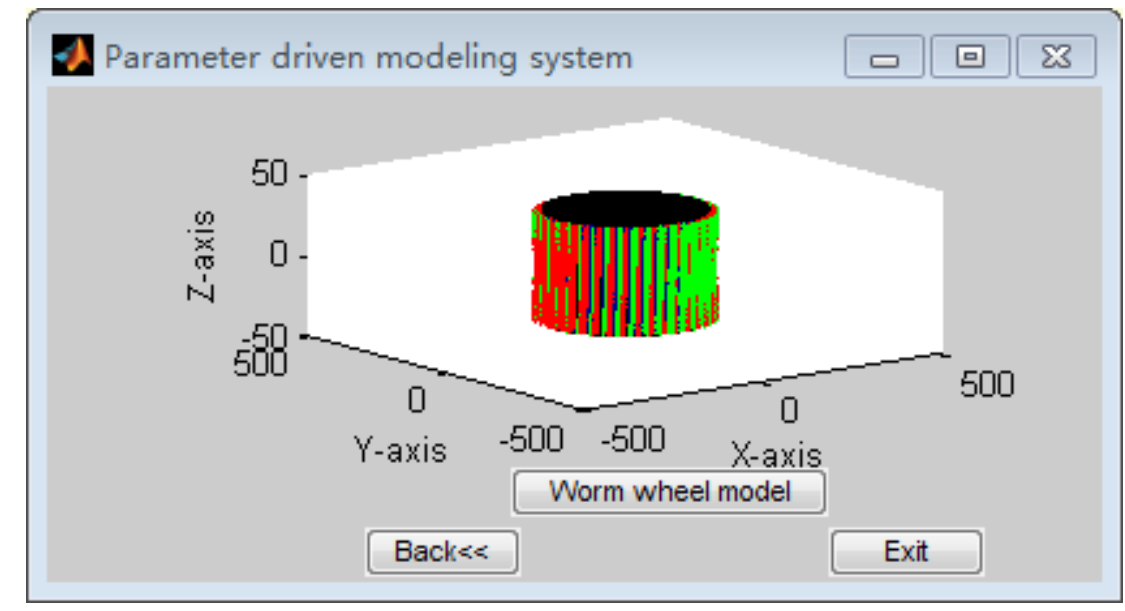

Fig.5 The model of the worm wheel

\section{Conclusion}

A parameter driven modeling method is proposed, in this method the parameters calculation and surface modeling are driven by the basic parameters of the worm. The parameter driven modeling system is developed applied MATLAB, the complex mathematical modeling operation is embedded in the related callback function, all of parameters calculation, meshing surface and 3D models are could obtained driven by the basic parameters for any size worm pair. The complex mathematical operations is simplified as well as the modeling efficiency and accuracy are also improved, this method has application and popularization significance.

\section{Acknowledgments}

This work is supported by the National Natural Science Foundation of China (NO.51275372), Hubei Province Science Foundation (NO.2016CFB148, NO.Q20162902), and School Science Project (NO.2015002003, NO.2015019703) 


\section{References}

[1] Dong Xue-zhu.The Design of Hourglass Worm and Its Modification [M]: Beijing: China Machine Press, 2004.

[2] Zhou Liang-yong. Hourglass Worm Modification Principle and Manufacturing Technology [M]. Changsha:National University of Defence Technology Press,2005.

[3] Ma long, Wu Xin-yue. Modeling of plane-generated double enveloping hourglass worm-gear pairs[J].Machinery Design\& Manufacture,2006,(6):62-64.

[4] Gao Hua-zhong, He Bang-gui. Modeling research of planar double-enveloping torus worm-drive [J].Machinery Design\&Manufacture, 2009, (3):130-132.

[5] Huang An-yi, Zhang zhong-fu, Huang Guan-pei. Research on AutoCAD-based 3D modeling and virtual machining for plane enveloping hourglass worm [J].Machinery Design\&Manufacture, 2002,(3):25-26.

[6] Huang Xiang-mao, Cao Xue-mei. Parameter optimization on plane-generated double enveloping torus worm pairs of the complex method[J].Machinery Design\&Manufacture,2014,(2):43-45.

[7] Tan Xin, Xu Lin-hong, and Zhou Hong. The optimized design of plane-generated double Enveloping torus worm pair based on the lubrication performance of preferred $[\mathrm{J}]$. Machinery Design\&Manufacture, 2005, (10):21-23.

[8] Zhi Liu, Hong Lu, Guoming Yu, Shaojun Wang. A novel CNC machining method for enveloping surface $[\mathrm{J}]$. International journal of advanced manufacturing technology, 2016, vol85:779-790. 